\title{
Development of Smartphone Applications for Nutrition and Physical Activity Behavior Change
}

Lana Hebden ${ }^{1}$, BND (Hons); Amelia Cook ${ }^{1}$, BAppSc/BSc (Hons); Hidde P van der Ploeg $^{2}$, PhD; Margaret Allman-Farinelli ${ }^{1}, \mathrm{PhD}, \mathrm{MPhilPH}$

\footnotetext{
${ }^{1}$ School of Molecular Bioscience, The University of Sydney, Sydney, Australia

${ }^{2}$ Sydney School of Public Health, The University of Sydney, Sydney, Australia
}

\section{Corresponding Author:}

Lana Hebden, BND (Hons)

School of Molecular Bioscience

The University of Sydney

Room 453, Level 4, Biochemistry and Microbiology Building G08

The University of Sydney

Sydney, 2006

Australia

Phone: 6193514672

Fax: 6190363184

Email: lana.hebden@sydney.edu.au

\section{Abstract}

Background: Young adults (aged 18 to 35) are a population group at high risk for weight gain, yet we know little about how to intervene in this group. Easy access to treatment and support with self-monitoring of their behaviors may be important. Smartphones are gaining in popularity with this population group and software applications ("apps") used on these mobile devices are a novel technology that can be used to deliver brief health behavior change interventions directly to individuals en masse, with potentially favorable cost-utility. However, existing apps for modifying nutrition or physical activity behaviors may not always reflect best practice guidelines for weight management.

Objective: This paper describes the process of developing four apps aimed at modifying key lifestyle behaviors associated with weight gain during young adulthood, including physical activity, and consumption of take-out foods (fast food), fruit and vegetables, and sugar-sweetened drinks.

Methods: The development process involved: (1) deciding on the behavior change strategies, relevant guidelines, graphic design, and potential data collection; (2) selecting the platform (Web-based versus native); (3) creating the design, which required decisions about the user interface, architecture of the relational database, and programming code; and (4) testing the prototype versions with the target audience (young adults aged 18 to 35 ).

Results: The four apps took 18 months to develop, involving the fields of marketing, nutrition and dietetics, physical activity, and information technology. Ten subjects provided qualitative feedback about using the apps. The slow running speed of the apps (due to a reliance on an active Internet connection) was the primary issue identified by this group, as well as the requirement to $\log$ in to the apps.

Conclusions: Smartphone apps may be an innovative medium for delivering individual health behavior change intervention en masse, but researchers must give consideration to the target population, available technologies, existing commercial apps, and the possibility that their use will be irregular and short-lived.

(JMIR Res Protoc 2012;1(2):e9) doi: 10.2196/resprot.2205

\section{KEYWORDS}

cellular phone; young adult; primary prevention; lifestyle; health behavior 


\section{Introduction}

Across developed countries, the average person owns 1.18 mobile phones with this number continuing to rise [1]. Much of this growth has been in smartphone ownership (mobile phones with computer and Internet capabilities); there were more than 490 million shipments of smartphones globally in 2011 compared to approximately 300 million in 2010 [2]. The growth in the smartphone market has been concentrated in young adults, especially in the United States, with $62 \%$ of mobile phone users aged 25-34 owning a smartphone in 2011, an increase from $41 \%$ in 2010 [3].

The development of smartphones has led to a proliferation of smartphone software applications ("apps"), which are programs able to run on these mobile devices. From a public health perspective, smartphone apps can potentially enhance the delivery of health behavior change interventions to individuals en masse and result in favorable cost-utility. Despite this, researchers to date have largely developed apps to intervene in the clinical care setting for patient self-management, whereby a patient monitors themselves and receives therapeutic feedback [4,5], or for real-time therapy where no self-reported data is required from the patient [6,7]. The commercial sector has developed numerous apps for weight loss that include information on nutrition and physical activity, although the majority are based on calorie counting approaches and may not always reflect best practice guidelines for weight management [8].

In most Western countries, young adults (ages 18 to 35) are a population group at high risk for becoming overweight or obese $[9,10]$. For example, in the US Coronary Artery Risk Development in Young Adults (CARDIA) cohort, it was reported that females gained an average $0.7 \mathrm{~kg}$ and males gained an average $0.8 \mathrm{~kg}$, each year [11]. There are four key lifestyle behaviors that appear to play an important role in the etiology of weight gain in this population group. These behaviors include a decline in physical activity $[12,13]$, excessive intake of high-fat take-out (fast food) meals [14], over-consumption of sugar-sweetened drinks [15,16], and an inadequate consumption of fruit and vegetables [17]. However, there is limited evidence to inform what method of intervention might be effective for preventing weight gain in this group [18], although easy access to treatment and providing support for planning and self-monitoring behavior may be important $[19,20]$. Hence, we embarked on building a series of smartphone apps to assist young adults in forming healthier lifestyle habits. This paper describes the process of developing four separate smartphone apps and discusses our insights from this process.

\section{Methods}

The development process consisted of four stages: (1) deciding on the specifications, (2) selecting the platform, (3) creating the design, and (4) testing the prototypes.

\section{Stage 1: Deciding on the Specifications}

The first stage of this process involved defining the purpose of each app. This required specifying the relevant public health guidelines to inform the goals for behavior change, the specific strategies for behavior change, the visuals or graphic design, and the potential data to be collected.

The fundamental purpose of the apps was to support change in the lifestyle behaviors identified. To assist young adults with improving their dietary habits, goals for these behaviors had to be defined to create rules about what is adequate [21,22]. Relevant public health guidelines were consulted for physical activity levels [23,24], intake of fruit and vegetables [25], and recommended limits for take-out meals and sugar-sweetened drinks [26,27]. For example, the physical activity app (ePASS) included a "good health" target of 30 minutes of moderate level physical activity daily based on the World Health Organization's physical activity guidelines of at least 150 minutes of aerobic activity per week for adults [24], and a "healthy weight" target of 60 minutes of moderate level physical activity daily based on expert consensus that up to 60 minutes of moderate activity daily is required to prevent unhealthy weight gain [23] (see Figure 1B). The fruit and vegetable app (eVIP) provided users with a graphical display of the number of fruit and vegetable servings they recorded out of the two servings of fruit and five servings of vegetables recommended by the Australian Government Department of Health and Aging (see Figure 1A) [25]. The sugar-sweetened drinks app (eSIYP) presented users with a color display of their total energy, sugar, and alcohol intake from all drinks consumed, in which green, orange, and red indicated the "ideal," "acceptable," and "too much" threshold levels of intake, respectively. These threshold levels were based on nutritional expert opinion of the recommended limits on consumption of added sugars, as per the Australian Dietary Guidelines [26]. The take-out food app (eTIYP) also presented users with a color display of the average energy and fat content of take-out meals, in which green indicated acceptable intake and red indicated excessive intake, equating to $\leq 30 \%$ and $>30 \%$ of the dietary intake recommended by the Australian National Health and Medical Research Council and the New Zealand Ministry of Health, respectively, according to age and gender [27].

In terms of behavior change strategies, young adults often lack the self-regulatory skills, such as self-monitoring and planning, required to adopt and maintain healthy behaviors [20]. Self-regulation was fostered in each app by providing users with a platform to create daily entries of their behavior (eg, physical activities performed or vegetables consumed) from which they were provided daily or weekly summaries of their reported behavior, in reference to public health guidelines, to enable their monitoring of and planning around these behaviors. Providing feedback that is personally relevant may also be an important strategy for changing young adults' behaviors [18]. Encouragement from success of attaining a goal and social persuasion can enhance self-efficacy, and has been identified as important in achieving behavior change, particularly during busy or stressful situations [28,29]. All apps provided motivational tips as a source of positive encouragement that would assist the young adults in creating more positive beliefs around their ability to change their behavior (eg, "You can split up your exercise target into as little as 15-minute bursts"). These tips were also tailored to users' self-reported behavior. For 
example, if a user's reported physical activity did not meet recommended guidelines, they were shown a relevant motivational tip (ie, "Plan exercise in advance and write it down if you can - try phone reminders").

Consideration was also given to the graphic design and how this might influence behavior. The behavior-image model suggests that through processes of social- and self-comparison, individuals will compare themselves to similar human images and create projections of themselves possessing the desired characteristics of the humans in those images [30]. This process is referred to as "self-reevaluation" in the Transtheoretical Model, which uses healthy role models and imagery to assist ones progression from contemplating behavior change to preparing for changing behavior [31]. Hence, in the apps we used images of young adults who were performing the target behaviors (eg, riding a bike or drinking water) and possessing desirable characteristics of a normal healthy appearance and lifestyle, to motivate users toward changing the target behaviors.
Similarly, we displayed healthier foods and drinks, rather than "junk" foods, to model these foods as ideal for consumption. All images were purchased from a commercial graphics company to avoid potential breaches in copyright.

To facilitate future research, we enabled the following data items to be exported: user identification (ID), log-in ID, sex, age, and the date and time of log-ins into each app. Additional information that was able to be exported from each app included: physical activities performed and their duration; drinks consumed and their volume, total sugar, alcohol, and energy content; energy and fat content of take-out meals, the restaurant where the meal was consumed, and the contents of the meal; and the number of servings and types of fruit and vegetables consumed. This data could be exported into comma-separated value files from our relational database (described later), which could then be exported into statistical software for further analysis.

Figure 1. Screenshots taken on an iPhone device illustrating the user interface: home screen provides an overview of fruit and vegetables recorded in eVIP (A) or physical activity recorded in ePASS (B), in light of reference population health guidelines.
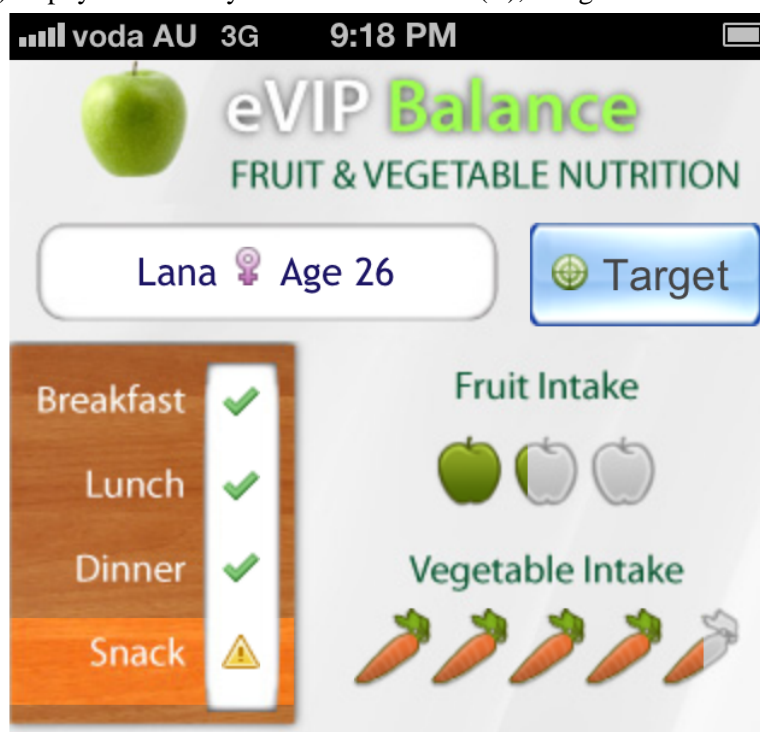

Fruit Intake

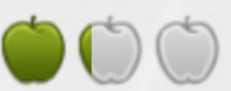

Vegetable Intake

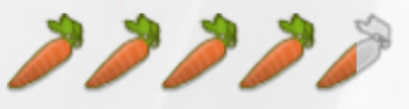

Didn't get 2Fruit \& 5Veg today? Fill a container with salad to have at lunch tomorrow.

\section{Record Serving}

View Recommended Recipes

(A)

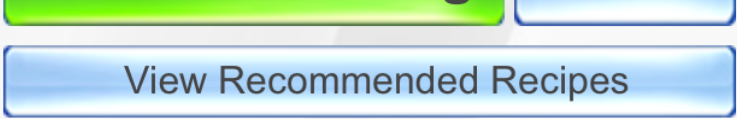

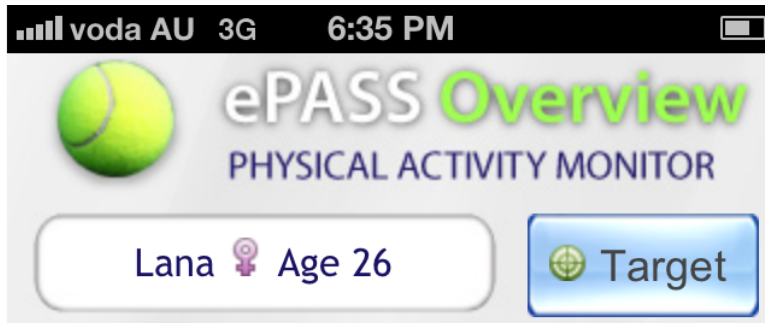

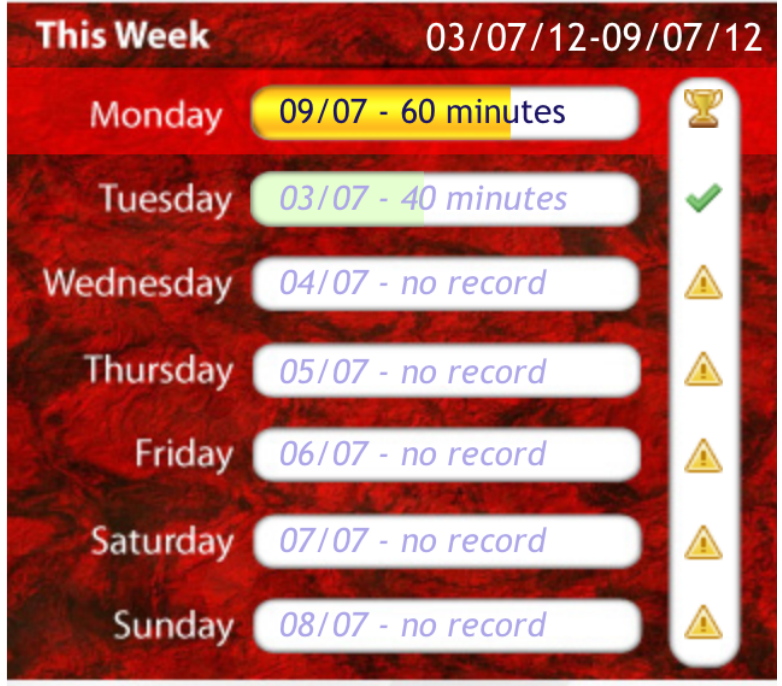

(B)

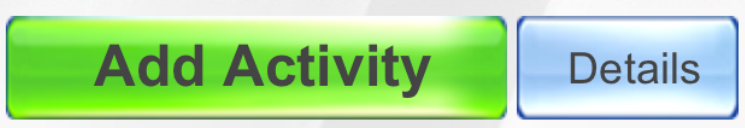

differences, Web-based apps may be used on all smartphones regardless of the operating system as long as the user has Internet access; however, there is less opportunity to utilize the existing hardware built into the phone (eg, the camera, geopositioning, or calendar). In our case, we developed Web-based apps because we did not require the use of existing hardware on the phone and we wanted to enable downloading of data recorded by users and allow the apps to be used on multiple operating systems and through the Internet for those who did not own a smartphone. However, Web-based apps are 
increasingly becoming able to perform like native apps with an offline mode that can be accessed without Internet connectivity. Similarly, there is increasing potential for native apps to possess Internet connectivity, enabling the user to download updates and data from the user to be uploaded to a server. This emphasizes the dynamic nature of mobile technologies and why the type of platform selected should be discussed with an information technology specialist.

\section{Stage 3: Creating the Design}

To enable users to record their behavior, each app had to be linked with the relevant data for that behavior. The following data items were included for each app:

1. ePASS: type of activity (ie, gym, sports, recreational, or housework) and the intensity (ie, moderate vs vigorous) of 91 unique activities, where "moderate" was defined as a metabolic equivalent of task (MET) value of 3-6 and "vigorous" was defined as $>6$, derived from the compendium of physical activities [32].

2. eVIP: serving size equivalents for 48 types of fruit and 61 vegetables, where 1 serving was equivalent to $150 \mathrm{~g}$ of fruit or $75 \mathrm{~g}$ of vegetables [26] (eg, 0.5 cups chopped or 4 spears of cooked asparagus are both equivalent to 1 serving of vegetables).

3. eTIYP: total energy and fat content of 504 take-out food and drink menu items.

4. eSIYP: drink category (eg, waters, vitamin waters, hot chocolate, tea/coffee, alcohol, soft drinks, sports drinks, cordials, fruit juices, fruit drinks, flavored milks, and milkshakes) and the total energy, sugar, and alcohol content of 114 unique drinks.

Nutrient composition data for eTIYP and eSIYP were sourced from the Australian government food and nutrient database, NUTTAB [33]. The foods listed in this database were chemically analyzed or, when unavailable, sourced from food manufacturer nutrient label data which may or may not be based on chemical analysis.

These data items were all contained within one relational database, which is essentially a database where the data items (or variables) are arranged into a series of tables with each table representing a different aspect or "relation" in the data. For each app, there were 1-2 tables containing the behavioral data. For example, the eVIP app required one table for the types of fruit and vegetables, such as "eggplant/aubergine (cooked)," and a second table listing the portion sizes, such as " 0.5 cups diced," "3 thin slices," or "1 thick slice," with their respective serving equivalents of 1 serving, 1 serving and 0.5 servings. An additional table was included for each app containing the motivational tips and another table contained user details (ie, name, age, gender, log-in ID, and user ID). The relational database management system software, MySQL (Oracle Corporation, Redwood Shores, California, United States), was used to access and query data items contained within the relational database using structured query language (SQL). For example, if a user logged into the eVIP app at lunchtime, SQL was used to identify a motivational tip about including fruit or vegetables at the lunch meal to present to the user from the motivational tips table in the relational database for the eVIP app.

Programming for the apps was written with Python programming language software (Python Software Foundation, Wolfeboro Falls, New Hampshire, United States) to communicate with the relational database and generate the user interface (ie, what the user sees and interacts with), including the HyperText Markup Language (HTML) (ie, the building blocks of a Web page) and the cascading style sheets (CSS) (ie, the visual formatting of the HTML). The HTML and CSS information was then interpreted by the Web browser on the user's mobile device to create the user interface (see Figures 1-5). To illustrate an example of this programming, if a user recorded 0.5 servings of fruit, this data was stored in the relational database and the user was instantly presented with an image of half of one apple shaded green (representing 0.5 servings of fruit) on the home screen of the eVIP app (see Figure 1A). A separate app was created for each of the four behaviors, rather than creating a combined app, to permit the targeting of different behaviors in future intervention research, such as addressing only those behaviors that are particularly challenging for the individual. Further, there was a need to limit each app to $<6$ screens, five of which were common to all of the apps (Figures 1-5), to simplify and increase the speed at which users could navigate their way through the apps.

\section{Stage 4: Testing the Prototypes}

Data presented and manipulated in the prototype version of each app were crosschecked against the relational database by two authors (LH and AC) for accuracy. This involved recording intake and activity behaviors at random in each of the four apps and checking whether the data presented to the users were correct (eg, the total energy of a take-out food item) and that the data were accurately manipulated in the apps (eg, converting fruit and vegetable portions recorded into the number of equivalent servings or summing the total energy content of all sugar-sweetened drinks recorded).

Twenty-one adults aged 18 to 35 years who were participating in a weight loss trial were provided access to the apps, and were asked for their feedback on the performance of the apps as part of an online survey. This survey included two questions assessing the usability of the apps, including: "Did you have any problems downloading the smartphone apps?" and "Did you have any problems using the smartphone apps?" with three response options: "yes," "no," or "did not access them." If they responded "yes," they were then prompted in an open-ended question: "Please tell us what problems you experienced." Two other open-ended questions asked, "How could the smartphone apps be improved?" and "Please tell us any other comments that you have." Re-occurring themes in the qualitative responses to these open-ended questions were identified and summarized. Procedures for collecting this information were approved by the University of Sydney Human Research Ethics Committee (approval \#13698). 


\section{Results}

The apps took 18 months to build including creation of the relational databases, exploring behavior change strategies, reviewing research with young adults on the key behaviors, creating the designs with information technology support, and testing the prototypes. Once the first app was developed, the others took less time with the physical activity app developed most rapidly. The development involved the fields of marketing, nutrition and dietetics, physical activity, and information technology. The cost of building all of the information into an app was approximately US $\$ 5000$ per app-less than half the cost of mainstream commercial companies because we employed Information Technology students for the development.

The four apps were found to return the correct data to the user from the relational database and calculations performed by the apps were accurate (eg, calculating the energy, sugar, and alcohol content in $390 \mathrm{~mL}$ of a beverage recorded by the user). The same generic user interface (ie, what the user sees and interacts with) was used in all four apps. Figures 1-5 present two examples (A and B) for each of the five generic screens of the user interface. The first screen users see when they launch one of the apps is a log-in screen (Figure 2), that requires users to enter a unique 4-digit log-in ID code. This was to protect the privacy of users (in the case that these apps were used in future human research), as well as the intellectual copyright of the university. The second screen seen by the users after they log in shows the home screen (Figure 1), that displays a summary of the user's behavior compared with reference guidelines. Screens presented in Figures 3 and 4 allow users to record their behaviors and review or edit data they have entered, respectively. Details about reference guidelines are then displayed in a "targets" screen (Figure 5).

Of the 21 participants offered to use these apps, only 10 evaluated them. These participants reported no difficulty with downloading the apps. Overall, participants did not like having to $\log$ in to use the apps. Some participants complained the apps operated slowly on their mobile devices (eg, "The smart phone app was a good idea but as it was a Web app, it often froze and it was a bit slow in general and scrolling lists were not functional on some operating systems" [from a 19-year-old female] and "Some parts of the apps didn't work for me, such as the scroll, so I couldn't enter many fruits/veg" [from a 22-year-old female]). Only one respondent (33-year-old female) provided a suggested improvement: "Applications could be designed to reward/monitor good behaviors only..."

Figure 2. Screenshots taken on an iPhone device illustrating the user interface: log-in screens where users enter their unique ID for user privacy and protection of intellectual property.

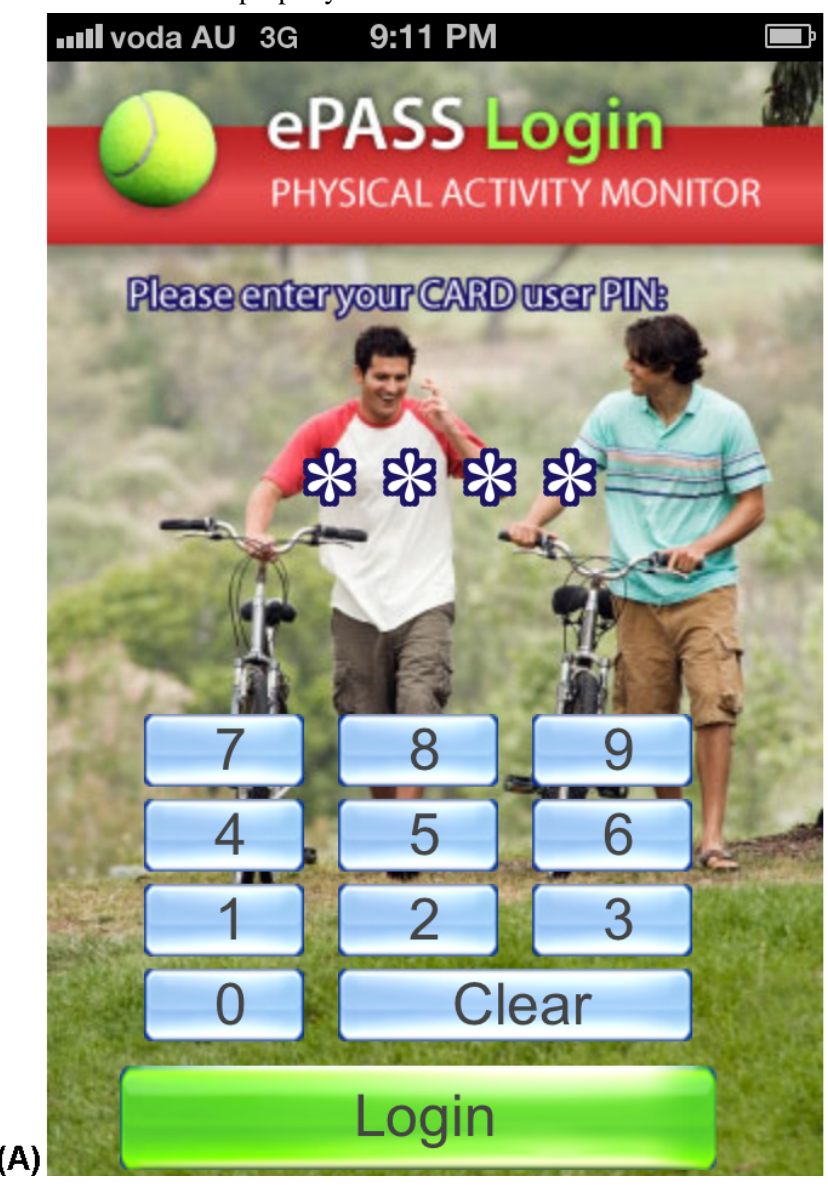

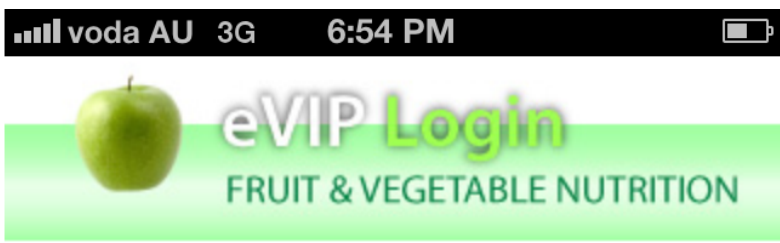

Please enter your CARD user PIN:
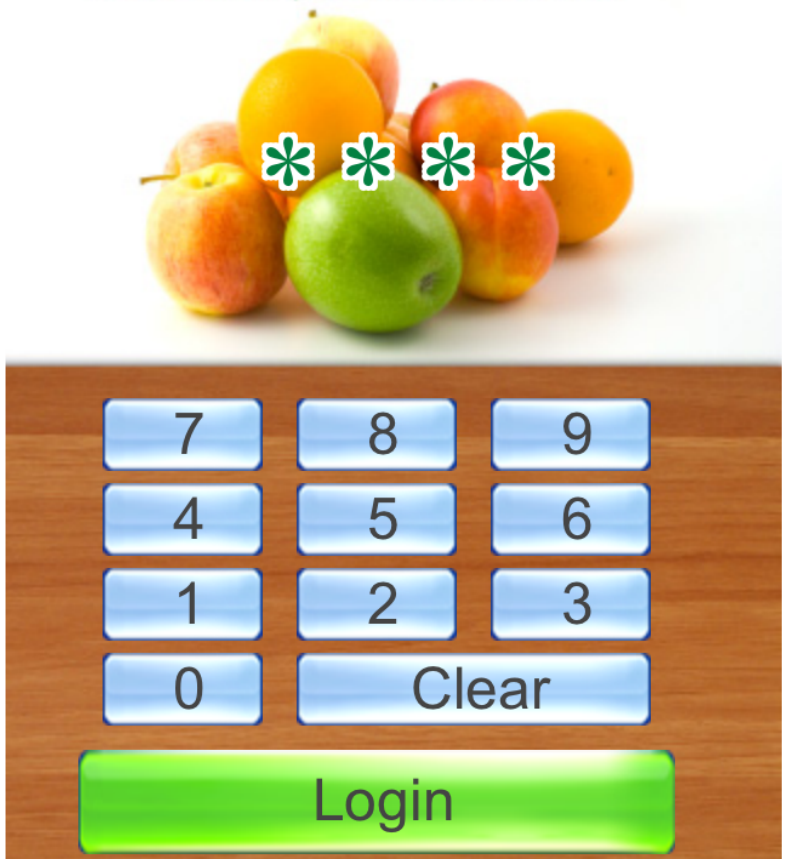
Figure 3. Screenshots taken on an iPhone device illustrating the user interface: users may record their take-out meals in eTIYP (A) or their drinks in eSIYP (B).

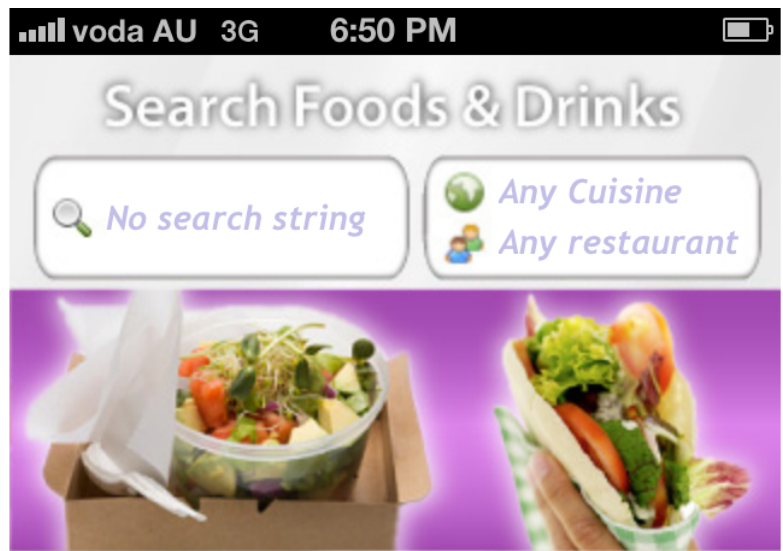

7Up (1 375mL can)

7Up (1 600mL bottle)

Aloo Muttar Potato \& Pea Curry (regular serve no rice)

Angry Angus (double)

Angry Angus (ingle)

Angus Wrap

Anzac cookie

(A)

\section{Record Item}

Cancel
(B)

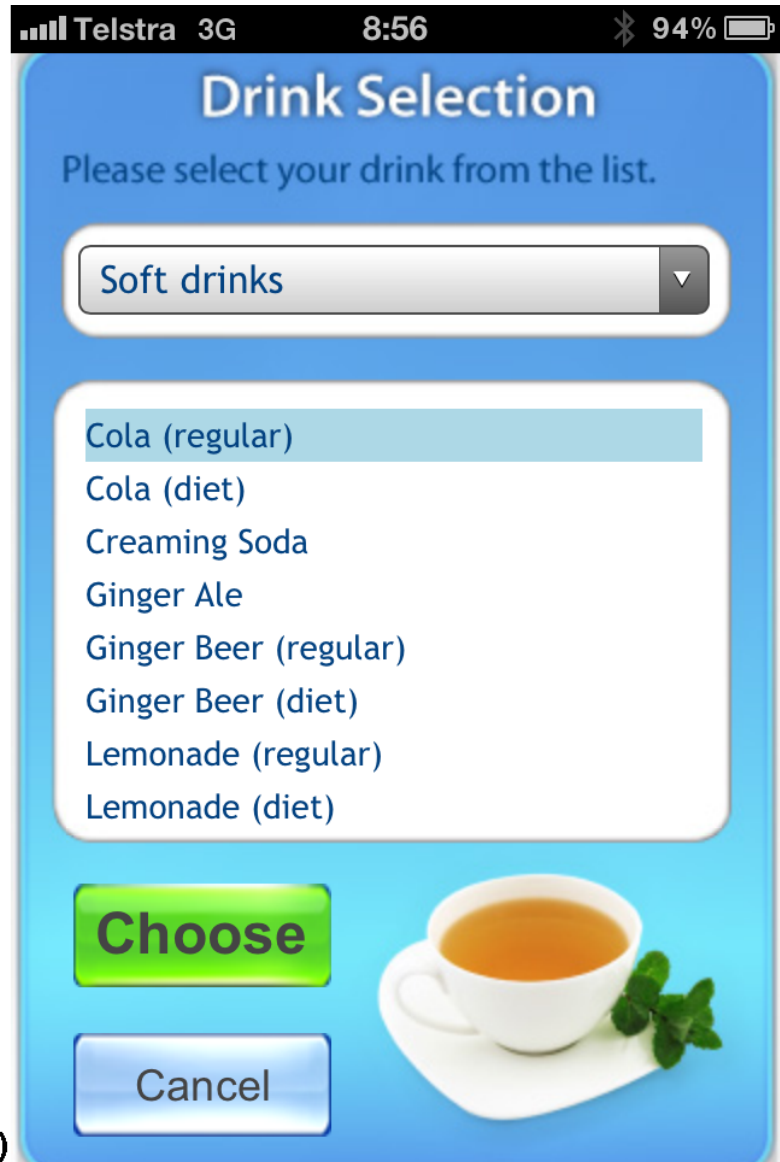


Figure 4. Screenshots taken on an iPhone device illustrating the user interface: users may review or edit their drinks in eSIYP (A) or their fruit and vegetable intake in eVIP (B).

\section{nlll voda $\mathrm{AU} 3 \mathrm{G}$}

\section{Edit Listi of Drinks}

Bottled, Non-Sparkling Water

$600 \mathrm{~mL}$

Cola (regular)

$600 \mathrm{~mL}$

Pre-Mixed Drinks (regular strength, 5\% alc.)

$310 \mathrm{~mL}$
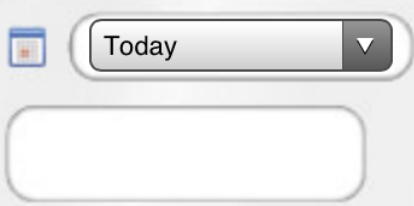

\section{Delete Selected}

(A)

\section{Edit Selected}

Done $\square$

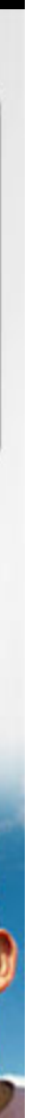

nnll voda AU 3G 9:19 PM $\square$

\section{View and Edit Servings}

Breakfast

Bananas 1 large

Lunch

Broccoli (cooked) 1 floret

Pumpkin (cooked) 1/4 cup

Fennel (raw) 1/4 cup sliced

Avocado 1/4 whole medium

Capsicum $1 / 2$ cup sliced raw or $1 / 4$ medium

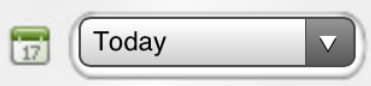

1.25 servings

Delete Selected

Edit Selected

Done

(B)

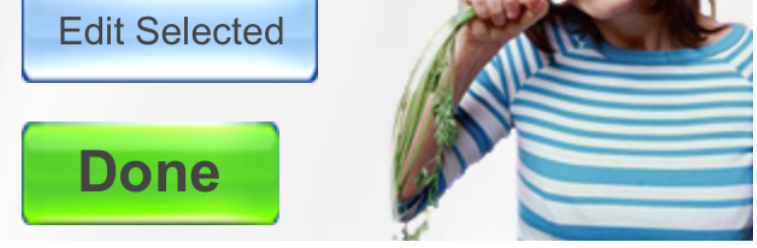


Figure 5. Screenshots taken on an iPhone device illustrating the user interface: targets screen provides the user details about the reference guidelines.

\section{nnllvoda $A U$ 3G 6:57 PM}

\section{eVMP TaMgefis}

FRUIT \& VEGETABLE NUTRITION
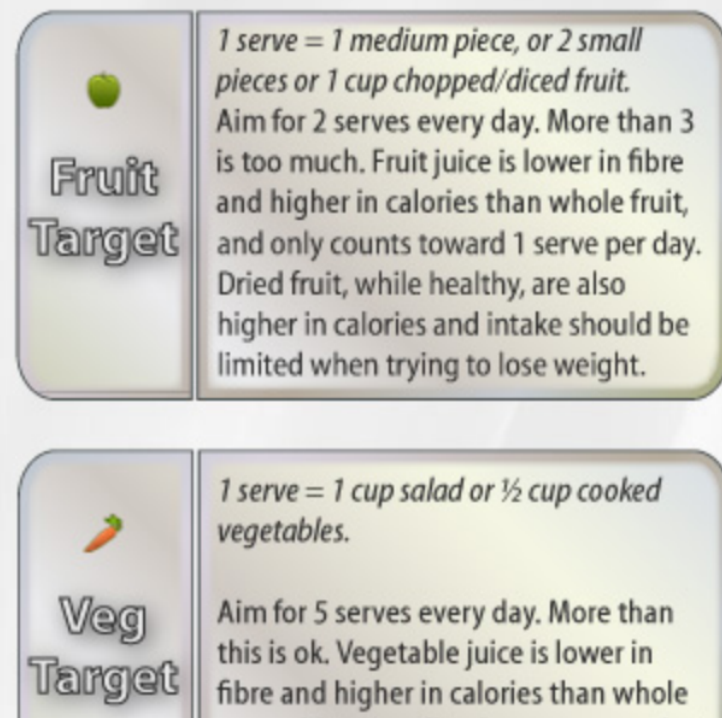

1 serve $=1$ cup salad or $1 / 2$ cup cooked vegetables.

Aim for 5 serves every day. More than this is ok. Vegetable juice is lower in fibre and higher in calories than whole vegetables, so will only count towards 1 serve per day.

(A)

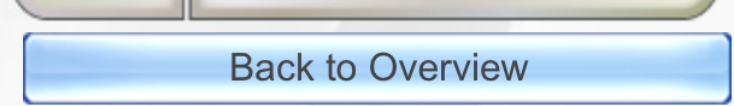

\section{Discussion}

In this paper, we have described the process of developing four smartphone apps aimed at improving nutrition and physical activity lifestyle behaviors during young adulthood. The apps were found to present precise data and to manipulate data accurately for the user. A small sample of young adults provided qualitative feedback. It was found that the slow running speed of the apps (because of their reliance on an active Internet connection) was an issue for the target audience (young adults aged 18 to 35 ), as was the requirement to $\log$ in to the apps. There was little suggestion for change in the information provided or graphics used in the apps, although most of the open-ended questions were posed to ask about issues or problems with the apps so that it generated negative rather than positive feedback. It is also acknowledged that the small sample size of participants testing the apps limits the validity of these findings.

Very few researchers in public health have reported on the development and use of smartphone apps for individual dietary or physical activity change. Mattila et al used a wellness diary for recording self-management of weight-related behaviors [34], Hughes et al developed an app for monitoring energy balance [35], Lee et al developed a weight loss diet game [36], while others have monitored diet or physical activity as part of a program for diabetes [37] or cardiac rehabilitation [38]. The uptake and usage of these apps has been moderate to high among adults in the intervention setting [34,36-38]. Smartphone apps have the potential to improve population health, largely because nnll voda AU 3G 6:35 PM

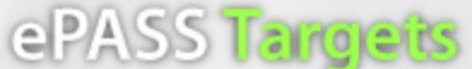 \\ PHYSICAL ACTIVITY MONITOR}

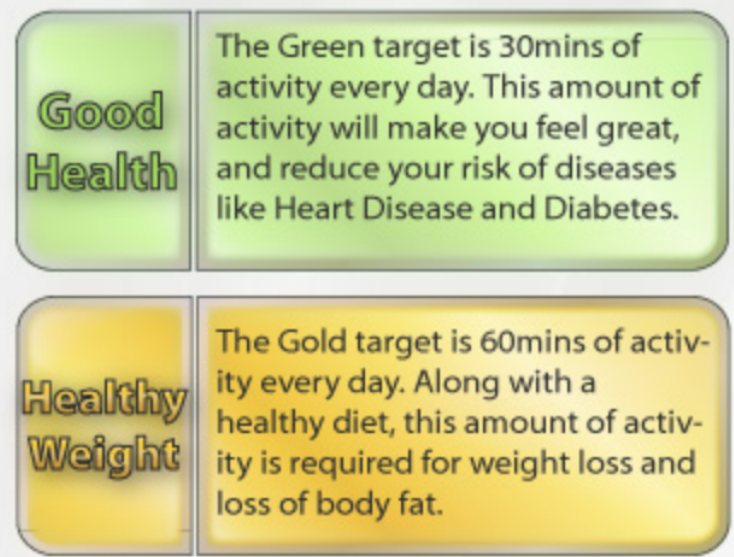

Some activities double $(x 2)$ your activity minutes as they are more intense, e.g. 20 mins walking uphill = $\mathbf{4 0}$ mins activity! You should always aim to increase your breathing and heart rate during activities.

(B)

\section{Back to Overview}

of their widespread and increasing use, dynamic technological advancements, ability to download updates, and use of existing features (eg, Internet access, geopositioning technology, as well as photo, video, and voice recording capabilities), and the potential for reducing intervention delivery costs. However, there are limitations to the use of smartphone apps, primarily because they may be expensive to develop and their use is often irregular and short-lived [34]. Therefore, if the target behaviors require commitment in the longer term, as is the case with nutrition and physical activity behaviors, additional support strategies may be required to prolong individuals' motivation to use the apps [34]. Also to consider, is the competition from new apps being developed. For this reason, an audit of existing apps is recommended to inform whether adequate apps are already available [8]. A further limitation is that other barriers to nutritional and physical activity behavior change perceived by young adults cannot be addressed, such as financial costs and aspects of their social and physical environments [39,40], although one can address personal barriers, such as time constraints [39-42] or lack of self-monitoring skills [20]. The equality of using apps as a public health strategy also remains questionable, and is likely to depend on the specific target group of interest.

Although young adults are increasingly using smartphones, use in other population groups is unclear. For this reason, formative research with the target population may be required for some groups, such as older adults, before embarking on developing apps for this demographic [43]. Future research should also examine how commercially developed apps for diet or physical 
activity are being used by different population groups to improve our understanding of how this technology may be used to support behavior change.

The feedback from trialing the apps with the target population (young adults aged 18 to 35 ) will be used to refine the prototype versions of the developed smartphone apps. Attempts will be made to increase the speed of the apps and ensure functionality on all mobile phone operating systems popular with young adults. The revised apps will then be formally tested for their "usability," which measures the ability of a software product to be understood, learned, used, and be attractive to the user, and will involve an analysis of the number of steps or time required to complete set tasks within the software [44]. Others have extended these methods to testing the usability of mobile phone apps, suggesting additional items for evaluation $[45,46]$.
The apps will be added as part of a multi-component randomized controlled trial in young adults, together with mobile phone text messaging and phone coaching calls, to evaluate their impact using validated measures of diet, physical activity, and anthropometrics.

Smartphone apps may be an innovative medium for delivering individual health behavior change intervention en masse. Researchers or health professionals considering developing an app in their area must give careful consideration to the target population in terms of their access, ability to adopt this form of intervention, and preferences regarding the design, the current technologies available for app development, existing commercial apps, and the possibility that their use will be irregular and short-lived.

\section{Acknowledgments}

We would like to thank Ms. Kristi Gander who provided data on fruit and vegetable serving sizes for the fruit and vegetable app, and to Mr. Liviu Constantinescu who with Dr. Peter Budd developed the apps, for his assistance with the information technology linguistics used in this paper.

\section{Authors' Contributions}

LH drafted the manuscript; LH, AC, and MA-F conceptualized the apps; HvdP informed the development of the ePASS (physical activity) app; AC, HvdP, and MA-F made revisions to the manuscript. All authors read and approved the final manuscript.

\section{Conflicts of Interest}

None declared.

\section{References}

1. ICT Data and Statistics Division, Telecommunication Development Bureau. Geneva, Switzerland: International Telecommunications Union. 2012 Jan. Mobile cellular subscriptions per 100 inhabitants, 2001-2011 (Excel Spreadsheet) URL: http://www.itu.int/ITU-D/ict/statistics/material/excel/2011/Mobile cellular 01-11.xls [accessed 2012-06-04] [WebCite Cache ID 68A4bzYe3]

2. International Data Corporation (IDC). Press Release. 2012 Feb. Smartphone market hits all-time quarterly high due to seasonal strength and wider variety of offerings, according to IDC (Press Release) URL: http://www.idc.com/getdoc. jsp?containerId=prUS23299912 [accessed 2012-06-04] [WebCite Cache ID 68A6LfCCi]

3. nielsen. Generation app: 62\% of mobile users 25-34 own Smartphones 2011 Nov (Press Release) URL: http://blog. nielsen.com/nielsenwire/online mobile/generation-app-62-of-mobile-users-25-34-own-smartphones/ [accessed 2012-06-04] [WebCite Cache ID 68A6QsNho]

4. Cummings E, Hauser J, Cameron-Tucker H, Fitzpatrick P, Jessup M, Walters EH, et al. Enhancing self-efficacy for self-management in people with cystic fibrosis. Stud Health Technol Inform 2011;169:33-37. [Medline: 21893709]

5. Klasnja P, Hartzler A, Powell C, Phan G, Pratt W. Health Weaver Mobile: Designing a Mobile Tool for Managing Personal Health Information during Cancer Care. AMIA Annu Symp Proc 2010;2010:392-396. [Medline: 21347007]

6. Burns MN, Begale M, Duffecy J, Gergle D, Karr CJ, Giangrande E, et al. Harnessing context sensing to develop a mobile intervention for depression. J Med Internet Res 2011;13(3):e55 [FREE Full text] [doi: 10.2196/jmir.1838] [Medline: 21840837]

7. Fletcher RR, Tam S, Omojola O, Redemske R, Kwan J. Wearable sensor platform and mobile application for use in cognitive behavioral therapy for drug addiction and PTSD. Conf Proc IEEE Eng Med Biol Soc 2011:1802-1805. [doi: 10.1109/IEMBS.2011.6090513] [Medline: 22254678]

8. Gan KO, Allman-Farinelli M. A scientific audit of smartphone applications for the management of obesity. Aust N Z J Public Health 2011 Jun;35(3):293-294. [doi: 10.1111/j.1753-6405.2011.00707.x] [Medline: 21627732]

9. Allman-Farinelli MA, Chey T, Bauman AE, Gill T, James WP. Age, period and birth cohort effects on prevalence of overweight and obesity in Australian adults from 1990 to 2000. Eur J Clin Nutr 2008 Jul;62(7):898-907. [doi: 10.1038/sj.ejcn.1602769] [Medline: 17440514]

10. Reither EN, Hauser RM, Yang Y. Do birth cohorts matter? Age-period-cohort analyses of the obesity epidemic in the United States. Soc Sci Med 2009 Nov;69(10):1439-1448. [doi: 10.1016/j.socscimed.2009.08.040] [Medline: 19773107] 
11. Norman JE, Bild D, Lewis CE, Liu K, West DS, CARDIA Study. The impact of weight change on cardiovascular disease risk factors in young black and white adults: the CARDIA study. Int J Obes Relat Metab Disord 2003 Mar;27(3):369-376. [doi: 10.1038/sj.ijo.0802243] [Medline: 12629565]

12. Hankinson AL, Daviglus ML, Bouchard C, Carnethon M, Lewis CE, Schreiner PJ, et al. Maintaining a high physical activity level over 20 years and weight gain. JAMA 2010 Dec 15;304(23):2603-2610. [doi: 10.1001/jama.2010.1843] [Medline: 21156948]

13. Leslie E, Fotheringham MJ, Owen N, Bauman A. Age-related differences in physical activity levels of young adults. Med Sci Sports Exerc 2001 Feb;33(2):255-258. [Medline: 11224815]

14. Pereira MA, Kartashov AI, Ebbeling CB, Van Horn L, Slattery ML, Jacobs DR, et al. Fast-food habits, weight gain, and insulin resistance (the CARDIA study): 15-year prospective analysis. Lancet 2005;365(9453):36-42. [doi: 10.1016/S0140-6736(04)17663-0] [Medline: 15639678]

15. Bleich SN, Wang YC, Wang Y, Gortmaker SL. Increasing consumption of sugar-sweetened beverages among US adults: 1988-1994 to 1999-2004. Am J Clin Nutr 2009 Jan;89(1):372-381 [FREE Full text] [doi: 10.3945/ajcn.2008.26883] [Medline: 19056548 ]

16. Duffey KJ, Gordon-Larsen P, Steffen LM, Jacobs DR, Popkin BM. Drinking caloric beverages increases the risk of adverse cardiometabolic outcomes in the Coronary Artery Risk Development in Young Adults (CARDIA) Study. Am J Clin Nutr 2010 Oct;92(4):954-959 [FREE Full text] [doi: 10.3945/ajcn.2010.29478] [Medline: 20702604]

17. Kimmons J, Gillespie C, Seymour J, Serdula M, Blanck HM. Fruit and vegetable intake among adolescents and adults in the United States: percentage meeting individualized recommendations. Medscape J Med 2009;11(1):26. [Medline: 19295947]

18. Hebden L, Chey T, Allman-Farinelli M. Lifestyle intervention for preventing weight gain in young adults: a systematic review and meta-analysis of RCTs. Obes Rev 2012 Aug;13(8):692-710. [doi: 10.1111/j.1467-789X.2012.00990.x] [Medline: 22413804]

19. Gokee LaRose J, Gorin AA, Clarke MM, Wing RR. Beliefs about weight gain among young adults: potential challenges to prevention. Obesity (Silver Spring) 2011 Sep;19(9):1901-1904. [doi: 10.1038/oby.2011.203] [Medline: 21738239]

20. Strong KA, Parks SL, Anderson E, Winett R, Davy BM. Weight gain prevention: identifying theory-based targets for health behavior change in young adults. J Am Diet Assoc 2008 Oct;108(10):1708-1715. [doi: 10.1016/j.jada.2008.07.007] [Medline: $\underline{18926139]}$

21. Maclellan DL, Gottschall-Pass K, Larsen R. Fruit and vegetable consumption: benefits and barriers. Can J Diet Pract Res 2004;65(3):101-105. [Medline: 15363114]

22. Walsh JR, White AA, Greaney ML. Using focus groups to identify factors affecting healthy weight maintenance in college men. Nutr Res 2009 Jun;29(6):371-378. [doi: 10.1016/j.nutres.2009.04.002] [Medline: 19628102]

23. Saris WH, Blair SN, van Baak MA, Eaton SB, Davies PS, Di Pietro L, et al. How much physical activity is enough to prevent unhealthy weight gain? Outcome of the IASO 1st Stock Conference and consensus statement. Obes Rev 2003 May;4(2):101-114. [Medline: 12760445]

24. World Health Organization. Global recommendations on physical activity for health. Geneva, Switzerland: World Health Organization; 2010.

25. Smith A, Kellett E, Schmerlaib Y. The Australian Guide to Healthy Eating: Background information for nutrition educators. Canberra, ACT: Commonwealth Department of Health and Family Services; 1998. URL: http://www.health.gov.au/internet/ main/publishing.nsf/content/FD699468D52A5A2ECA256F19000406D6/\$File/fdeduc.pdf [accessed 2012-08-20] [WebCite Cache ID 6A3Q1gUYt]

26. National Health and Medical Research Council. Food for health: Dietary guidelines for Australians. Canberra, ACT: Commonwealth Department of Health and Ageing; 2003.

27. National Health and Medical Research Council and New Zealand Ministry of Health. Nutrient Reference Values for Australia and New Zealand including recommended dietary intakes. Canberra, ACT: Commonwealth Department of Health and Aging; 2006. URL: http://www.nhmrc.gov.au/ files nhmrc/publications/attachments/n35.pdf [accessed 2012-08-20] [WebCite Cache ID 6A3QkqMOi]

28. de Bruijn GJ. Understanding college students' fruit consumption. Integrating habit strength in the theory of planned behaviour. Appetite 2010 Feb;54(1):16-22. [doi: 10.1016/j.appet.2009.08.007] [Medline: 19712718]

29. Horacek TM, White A, Betts NM, Hoerr S, Georgiou C, Nitzke S, et al. Self-efficacy, perceived benefits, and weight satisfaction discriminate among stages of change for fruit and vegetable intakes for young men and women. J Am Diet Assoc 2002 Oct;102(10):1466-1470. [Medline: 12396169]

30. Werch CC. The Behavior-Image Model: a paradigm for integrating prevention and health promotion in brief interventions. Health Educ Res 2007 Oct;22(5):677-690 [FREE Full text] [doi: 10.1093/her/cyl146] [Medline: 17138616]

31. Prochaska JO, DiClemente CC, Norcross JC. In search of how people change. Applications to addictive behaviors. Am Psychol 1992 Sep;47(9):1102-1114. [Medline: 1329589]

32. Ainsworth BE, Haskell WL, Herrmann SD, Meckes N, Bassett DR, Tudor-Locke C, et al. 2011 Compendium of Physical Activities: a second update of codes and MET values. Med Sci Sports Exerc 2011 Aug;43(8):1575-1581. [doi:

10.1249/MSS.0b013e31821ece12] [Medline: 21681120] 
33. NUTTAB 2010 - Food Composition Tables (Online Database).: Food Standards Australia New Zealand; 2011. URL: http:/ /www.foodstandards.gov.au/consumerinformation/nuttab2010/ [accessed 2012-08-02] [WebCite Cache ID 69cyjCey4]

34. Mattila E, Korhonen I, Salminen JH, Ahtinen A, Koskinen E, Särelä A, et al. Empowering citizens for well-being and chronic disease management with wellness diary. IEEE Trans Inf Technol Biomed 2010 Mar;14(2):456-463. [doi: 10.1109/TITB.2009.2037751] [Medline: 20007055]

35. Hughes DC, Andrew A, Denning T, Hurvitz P, Lester J, Beresford S, et al. BALANCE (Bioengineering Approaches for Lifestyle Activity and Nutrition Continuous Engagement): developing new technology for monitoring energy balance in real time. J Diabetes Sci Technol 2010 Mar;4(2):429-434 [FREE Full text] [Medline: 20307404]

36. Lee W, Chae YM, Kim S, Ho SH, Choi I. Evaluation of a mobile phone-based diet game for weight control. J Telemed Telecare 2010 Jul;16(5):270-275. [doi: 10.1258/jtt.2010.090913] [Medline: 20558620]

37. Arsand E, Tatara N, Østengen G, Hartvigsen G. Mobile phone-based self-management tools for type 2 diabetes: the few touch application. J Diabetes Sci Technol 2010 Mar;4(2):328-336 [FREE Full text] [Medline: 20307393]

38. Varnfield M, Karunanithi MK, Särelä A, Garcia E, Fairfull A, Oldenburg BF, et al. Uptake of a technology-assisted home-care cardiac rehabilitation program. Med J Aust 2011 Feb 21;194(4):S15-S19. [Medline: 21401482]

39. Andajani-Sutjahjo S, Ball K, Warren N, Inglis V, Crawford D. Perceived personal, social and environmental barriers to weight maintenance among young women: A community survey. Int J Behav Nutr Phys Act 2004 Dec 5;1(1):15 [FREE Full text] [doi: 10.1186/1479-5868-1-15] [Medline: 15462679]

40. Greaney ML, Less FD, White AA, Dayton SF, Riebe D, Blissmer B, et al. College students' barriers and enablers for healthful weight management: a qualitative study. J Nutr Educ Behav 2009;41(4):281-286. [doi: 10.1016/j.jneb.2008.04.354] [Medline: 19508934]

41. Larson NI, Nelson MC, Neumark-Sztainer D, Story M, Hannan PJ. Making time for meals: meal structure and associations with dietary intake in young adults. J Am Diet Assoc 2009 Jan;109(1):72-79. [doi: 10.1016/j.jada.2008.10.017] [Medline: 19103325]

42. Welch N, McNaughton SA, Hunter W, Hume C, Crawford D. Is the perception of time pressure a barrier to healthy eating and physical activity among women? Public Health Nutr 2009 Jul;12(7):888-895. [doi: 10.1017/S1368980008003066] [Medline: 18647424]

43. Lorenz A, Oppermann R. Mobile health monitoring for the elderly: designing for diversity. PMC 2009;5(5):478-495. [doi: 10.1016/j.pmcj.2008.09.010]

44. Nielsen J. Usability engineering. San Francisco, California: Morgan Kaufmann Publishers; 1993.

45. Coursaris CK, Kim DJ. A meta-analytical review of empirical mobile usability studies. JUS 2011;6(3):117-171.

46. Zhang D, Adipat B. Challenges, methodologies, and issues in the usability testing of mobile applications. Int J Hum-Comput Int 2005;18(3):293-308. [doi: 10.1207/s15327590ijhc1803 3]

\author{
Abbreviations \\ app: smartphone application \\ CARDIA: Coronary Artery Risk Development in Young Adults \\ CSS: cascading style sheets \\ ePASS: physical activity smartphone app \\ eSIYP: sugar-sweetened drinks smartphone app \\ eTIYP: take-out (fast food) smartphone app \\ eVIP: fruit and vegetable smartphone app \\ HTML: HyperText Markup Language \\ ID: identification \\ MET: metabolic equivalent of task \\ SQL: structured query language
}

Edited by G Eysenbach; submitted 04.06.12; peer-reviewed by T Burrows, J Thomas; comments to author 26.06.12; revised version
received 10.07.12; accepted 02.08.12; published 22.08 .12
Please cite as:
Hebden L, Cook A, van der Ploeg HP, Allman-Farinelli $M$
Development of Smartphone Applications for Nutrition and Physical Activity Behavior Change
JMIR Res Protoc 2012;1(2):e9
URL: $\underline{\text { http://www.researchprotocols.org/2012/2/e9/ }}$
doi: $\underline{10.2196 / \text { resprot.2205 }}$
PMID: $\underline{23611892}$


CLana Hebden, Amelia Cook, Hidde P. van der Ploeg, Margaret Allman-Farinelli. Originally published in JMIR Research Protocols (http://www.researchprotocols.org), 22.08.2012. This is an open-access article distributed under the terms of the Creative Commons Attribution License (http://creativecommons.org/licenses/by/2.0/), which permits unrestricted use, distribution, and reproduction in any medium, provided the original work, first published in JMIR Research Protocols, is properly cited. The complete bibliographic information, a link to the original publication on http://www.researchprotocols.org, as well as this copyright and license information must be included. 